\title{
Multiple strokes after initiation of steroid therapy in giant cell arteritis
}

\author{
Julio Collazos, Carlos García-Moncó ${ }^{1}$, Aitor Martín, Jose Rodriguez, \\ Ma Angeles Gómez ${ }^{1}$
}

\author{
Sections of Internal Medicine and ${ }^{1}$ Neurology, Hospital de Galdácano, 48960 Vizcaya, Spain
}

\begin{abstract}
Summary: We report the case of a 66 year old woman with clinical manifestations of giant-cell arteritis, positive temporal artery biopsy and excellent response to prednisone, who developed massive cerebral infarcts after 5 days of treatment. Computed tomographic and magnetic resonance imaging scans revealed multiple infarcts in areas irrigated by both the carotid and vertebrobasilar systems bilaterally. $A$ four-vessel angiography performed 19 days later revealed only mild atheromatous disease. Anticardiolipin antibodies were negative and a transoesophageal echocardiogram was normal.

Stroke during the first 2 weeks of therapy in giant-cell arteritis, especially in the vertebrobasilar territory, has been previously reported. Anticoagulation during the first weeks of steroid treatment in these patients might be advisable.
\end{abstract}

\section{Introduction}

First described by Horton et al. in 1932, ${ }^{1}$ giant cell arteritis (GCA) has been recognized as an inflammatory condition that affects principally the extracranial vessels. ${ }^{2}$ Central nervous system (CNS) disease is uncommon, probably because GCA affects vessels that contain internal elastic lamina and intracranial vessels lose this lamina when they penetrate into the cranium. ${ }^{3}$ Moreover, some cases of CNS involvement attributed to GCA have probably been due to primary angiitis of the CNS rather than to $\mathrm{GCA}^{4}$ and the age group of patients with GCA predisposes them to atherothrombotic disease. Thus the actual frequency and severity of intracranial involvement is unknown.

In a review of 166 cases of biopsy-proven GCA, neurological problems occurred in 51 patients $(31 \%)$, a frequency higher than that reported in most other series, with only five patients $(3 \%)$ having strokes, ${ }^{5}$ a rate that may not substantially exceed the expected frequency for age-matched people. ${ }^{6}$ Stroke was also observed in two of 175 patients $(1.1 \%)$ in another series. ${ }^{7}$ We report the case of a patient with typical, histologically proven GCA, who developed multiple cerebral infarcts a few days after the initiation of steroid therapy.

\section{Case report}

A 66 year old woman in prior good health and with no past history of vascular risk factors was admit-

Correspondence: J. Collazos, M.D.

Accepted: 2 August 1993 ted to our hospital after suffering for 10 weeks from fever, sweats, anorexia, malaise, weight loss, diffuse headache, and pain and stiffness in shoulders, hips and thighs. She denied visual disturbances, scalp tenderness or jaw or tongue claudication. Physical examination was unremarkable except for thickened and tender temporal arteries bilaterally, and proximal muscle weakness. Serum chemistry was normal except for slightly increased alkaline phosphatase. Haematocrit was $30.2 \%$, leucocytes $9.8 \times$ $10^{9} / 1$ and platelet count $596 \times 10^{9} / 1$. Erythrocyte sedimentation rate (ESR) was $122 \mathrm{~mm}$ /hour. A right temporal biopsy was performed and prednisone $60 \mathrm{mg} /$ day was started with dramatic improvement in less than 24 hours. Examination of the biopsy material revealed a granulomatous, giant-cell arteritis with prominent inflammatory infiltrates affecting the internal elastic lamina and intimal fibrosis. A diagnosis of temporal arteritis with polymyalgia rheumatica was made and the patient was discharged after 3 days of steroid therapy, taking $40 \mathrm{mg} / \mathrm{day}$ of prednisone.

Two days later she developed acute bilateral loss of vision followed by confusion and progressive obtundation. Physical examination revealed a stuporous woman with bilateral blindness, normal pupillary reflexes and paresis of the left arm. Fundoscopy showed normal optic discs and small retinal haemorrhages bilaterally consistent with vasculitis. A computed tomographic (CT) scan obtained a few hours after the episode showed only calcification of the basal ganglia. The haematocrit was $36.1 \%$ and the ESR $98 \mathrm{~mm} /$ hour. A CT scan repeated 3 days later disclosed multiple infarcts 
affecting both occipital lobes, centrum semiovale bilaterally and left internal capsule. Magnetic resonance imaging (MRI) disclosed large infarcts in both occipital and left parietal lobe, and multiple smaller infarcts in corona radiata bilaterally, both centrum semiovale, internal and external capsule, pons and cerebellar peduncles (Figure 1). Lumbar puncture yielded a clear, acellular cerebrospinal fluid with normal chemistry values. An electrocardiogram and a transoesophageal echocardiogram were normal. Rheumatoid factor, anti-nuclear antibodies, anti-cardiolipin antibodies and antineutrophil cytoplasmic antibodies were negative. Visual evoked potentials gave normal results bilaterally. Four-vessel angiography performed 19 days after the stroke disclosed only mild atheromatous disease, with no evidence of thrombosis, significant stenosis or arteritis, either intracranially or extracranially. On the following weeks obtundation resolved, arm weakness significantly improved and the patient was left with an incomplete Balint's syndrome, showing optic ataxia and ocular apraxia but no simultagnosia. She was discharged on $40 \mathrm{mg}$ daily of prednisone, with no further complications up to now, 2 months after the onset.

\section{Discussion}

Stroke, albeit rare, is a grave and sometimes fatal complication of GCA. Our patient had bilateral

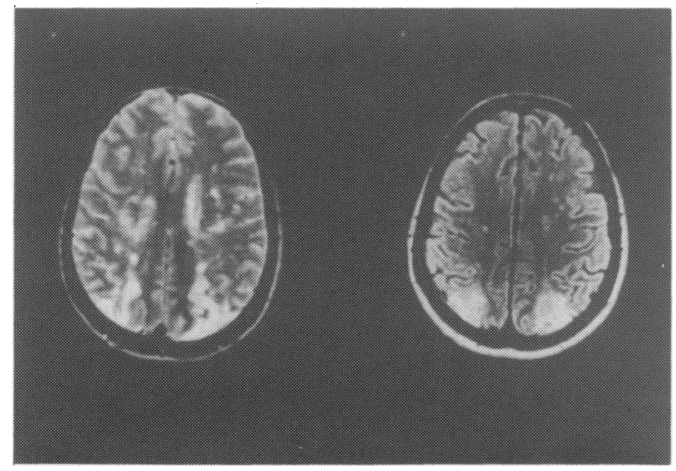

Figure $1 T_{2}$-weighted MRI showing bilateral occipitoparietal infarcts and subcortical white matter ischaemic lesions in the distribution of both carotid arteries. infarction in the distribution of the posterior cerebral arteries. This is most often due to occlusion of the basilar artery by embolic material from proximal atheroma. Although this mechanism cannot be completely ruled out, it would hardly explain the occurrence of infarcts in other arterial territories as happened in this patient. In the present case, we believe that stroke was related to GCA because no vascular risk factors were present other than age, there was a clear diagnosis of active GCA, the ictus followed the initiation of steroid therapy and multiple infarcts occurred simultaneously with no evidence of cardiac or aortic thrombi on transoesophageal echocardiography. Angiography did not show significant stenosis at any level, so arteritic involvement of the extracranial portion of carotid and vertebrobasilar systems seems the most likely mechanism, and in fact this has been the finding in autopsy-proven cases.

A study found the ratio of patients with carotid to those with vertebrobasilar infarctions to be $3: 2,{ }^{5}$ lower than the 5:1 ratio observed in the general population. ${ }^{8}$ Probably a more frequent involvement of the vertebral arteries as compared with other great vessels in the head and neck justify these differences. ${ }^{3}$

It is of general agreement that the presence of neurological disease is an indication for immediate steroid therapy in GCA. However, it is worrisome to note that several patients have been described who had stroke despite therapy with steroids, especially within 2 weeks of initiation of treatment. ${ }^{5,9}$ On the other hand, anti-cardiolipin antibodies are present in many patients with GCA and, in some of them, they have been associated with severe vascular complications. ${ }^{10}$ Accordingly, anticoagulant therapy could be recommendable in the management of these patients, especially in the first weeks of steroid treatment. However, our patient did not have anti-cardiolipin antibodies and yet she developed extensive infarcts.

Thus, no biological or clinical markers allow us to select reliably those patients who will develop brain infarction. Perhaps prospective analysis of large series will shed light on the detection and most appropriate management of patients prone to stroke in GCA.

\section{References}

1. Horton, B.T., Magath, T.B. \& Brown, G.E. An undescribed form of arteritis of the temporal vessels. Mayo Clin Proc 1932, 7: 700-701.

2. Goodman, B.W., Jr. Temporal arteritis. Am J Med 1979, 67: 839-852.

3. Wilkinson, I.M.S. \& Russell, R.W.R. Arteries of the head and neck in giant cell arteritis: a pathological study to show the pattern of arterial involvement. Arch Neurol 1972, 27: $378-391$.
4. Reich, K.A., Giansiracusa, D.F. \& Strongwater, S.L. Neurologic manifestations of giant cell arteritis. Am J Med 1990, 89: 67-72.

5. Caselli, R.J., Hunder, G.G. \& Whisnant, J.P. Neurologic disease in biopsy-proven giant cell (temporal) arteritis. Neurology 1988, 38: 352-359.

6. Garraway, W.M., Whisnant, J.P. \& Drury, I. The continuing decline in the incidence of stroke. Mayo Clin Proc 1983, 58: $520-523$. 
7. Hollenhorst, R.W., Brown, J.R., Wagener, H.P. \& Shick, R.M. Neurologic aspects of temporal arteritis. Neurology 1960, 10: 490-498.

8. Turney, T.M., Garraway, W.M. \& Whisnant, J.P. The natural history of hemispheric and brainstem infarction in Rochester, Minnesota. Stroke 1984, 15: 790-794.

9. Petty, G.N. \& Mohr, J.P. Stroke in the setting of collagen vascular disease. In: Barnett, H.J.M., Mohr, J.P., Stein, B.M. \& Yatsu, F.M. (eds) Stroke, Pathophysiology, Diagnosis and Management, 2nd edn. Churchill Livingstone, New York, 1992, pp. 691-720.
10. Espinoza, L.R., Jara, L.J., Silveira, L.H. et al. Anticardiolipin antibodies in polymyalgia rheumatica-giant cell arteritis: association with severe vascular complications. Am J Med 1991, 90: 474-478. 